\title{
A REPRESENTATION THEOREM FOR HARMONIC FUNCTIONS IN THE BALL IN $\mathbf{R}^{n}$
}

\author{
KRZYSZTOF SAMOTIJ
}

\section{Introduction}

Throughout this paper $n$ is an integer greater than or equal to 2 , and $B=\left\{x \in \mathbf{R}^{n}:|x|<1\right\}$ is the open unit ball in the $n$-dimensional Euclidean space $\mathbf{R}^{n}$, where $|x|$ denotes the Euclidean norm of $x \in \mathbf{R}^{n} . S=\left\{x \in \mathbf{R}^{n}:|x|=1\right\}$ is the Euclidean boundary of $B$. For arbitrary nonzero $x, y \in \mathbf{R}^{n}$ set

$$
\varphi(x, y)=\arccos \frac{x \cdot y}{|x||y|},
$$

where $x \cdot y$ stands for the inner product of $x$ and $y$. We denote by $P$ the Poisson kernel for $B$, i.e.,

$$
P(x, \eta)=\frac{1-|x|^{2}}{|x-\eta|^{n}}=\frac{1-|x|^{2}}{\left[1-2|x| \cos \varphi(x, \eta)+|x|^{2}\right]^{n / 2}}=\tilde{P}(|x|, \varphi(x, \eta)),
$$

whenever $x \in B$ and $\eta \in S$. For $\eta \in S$ and $t \geqq 0$ the set

$$
C(\eta, t)=\{\xi \in S: \varphi(\eta, \xi)<t\}
$$

will be referred to as an open spherical cap of radius $t$ centered at $\eta$, while the set

$$
\bar{C}(\eta, t)=\{\xi \in S: \varphi(\eta, \xi) \leqq t\}
$$

will be called a closed spherical cap of radius $t$ centered at $\eta . \sigma$ denotes the surface measure on $S$ so normalized that $\sigma(S)=1$. If $\mu$ is a finite Borel measure on $S$, then the Poisson integral of $\mu$ is defined, as usual, by

$$
P[d \mu](x)=\int P(x, \eta) d \mu(\eta), \quad x \in B .
$$

When $f \in L^{1}(\sigma)$, we write $P[f]$ in place of $P[f d \sigma]$. It is classical (and known in the case of $n=2$ as the Riesz-Herglotz theorem; see also [2], p. 175) that if $u \leqq 0$ is a harmonic function on $B$, then $u=P[d \mu]$ for some finite nonpositive Borel measure $\mu$ on $S$, called the boundary measure of $u$. In this case, for each $t \in[0, \pi]$ and $\eta \in S$ the limit

$$
u_{\eta}(t)=\lim _{r \rightarrow 1-} \int_{C(\eta, t)} u(r \xi) d \sigma(\xi)
$$


exists and

$$
u_{\eta}(t)=\frac{1}{2}[\mu(C(\eta, t))+\mu(\bar{C}(\eta, t))] \text { for each } t \in(0, \pi) .
$$

For each $x \in B$ we then have

$$
u(x)=\int_{0}^{\pi} \tilde{P}(|x|, t) d u_{\eta}(t) \text {, with } \eta=x /|x| \text { if } \quad x \neq 0,
$$

and with arbitrary $\eta \in S$ if $x=0$.

In this paper we prove the sufficiency of a certain condition on $u$ for the existence of the limits (1) and for the validity of the representation (3), even when there is hardly a trace of a boundary measure left. Namely, we will prove

Theorem 1. Let $u$ be a real harmonic function on $B$. Let $m(r)=\max _{\eta \in S} u(r \eta)$, $0 \leqq r<1$, and suppose that

$$
J=J(m)=\int_{0}^{1}\left[\frac{m(r)-m(0)}{1-r}\right]^{(n-1) / n} d r<\infty .
$$

Then the limit $u_{\eta}(t)$ defined by (1) exists for each $\eta \in S$ and each $t \in[0, \pi]$. Each $u_{\eta}$ as a function defined on $[0, \pi]$ has one-sided limits at each $t \in[0, \pi]$ and

$$
u_{\eta}(t)=\frac{1}{2}\left[u_{\eta}(t+)+u_{\eta}(t-)\right] \text { for each } t \in(0, \pi) .
$$

Moreover, the representation (3) is valid for every $x \in B$.

If we assume in addition that $u(0)=0$, then there exists a continuous increasing function $\varkappa$ on $[0, \pi]$ depending only on $m$ and $n$, with $\varkappa(0)=0$ and $\varkappa(\pi)=A_{0} J^{n /(n-1)}$, where $A_{0}$ is an absolute constant depending only on $n$, such that

$$
u_{\eta}\left(t_{2}\right)-u_{\eta}\left(t_{1}\right) \leqq \varkappa\left(t_{2}-t_{1}\right)
$$

whenever $0 \leqq t_{1}<t_{2} \leqq \pi$, and $\eta \in S$.

In the case where $n=2$ we can express our result in an apparently stronger form, as

Theorem 2. Let $u(z)$ be a real harmonic function in $|z|<1$. Let $m(r)=$ $\max _{|z|=r} u(z), 0 \leqq r<1$, and suppose that

$$
J=\int_{0}^{1} \sqrt{\frac{m(r)-m(0)}{1-r}} d r<\infty .
$$

Then

(i) for each arc I of the unit circle the limit

$$
\mu(I)=\lim _{r \rightarrow 1-} \frac{1}{2 \pi} \int_{I} u(r z)|d z|
$$

exists,

(ii) $\left(6^{\prime}\right)$

$$
\mu(I) \leqq \varkappa\left(\frac{|I|}{2}\right)
$$

provided $u(0)=0$, where $x$ is as in Theorem 1 and $|I|$ denotes the length of $I$, 
(iii) if $\tilde{\mu}(t)=\mu\left(\left\{e^{i \theta}: 0 \leqq \theta \leqq t\right\}\right)$, all discontinuities of $\tilde{\mu}$ are of the first kind, and moreover

$$
\tilde{\mu}(t)=\frac{1}{2}[\tilde{\mu}(t-)+\tilde{\mu}(t+)], \quad \text { if } \quad t \in(0,2 \pi),
$$

and

$$
\tilde{\mu}(0+)=\tilde{\mu}(2 \pi)-\tilde{\mu}(2 \pi-),
$$

(iv) the following representation is valid:

$$
u(z)=\int_{0}^{2 \pi} \frac{1-|z|^{2}}{\left|e^{i \theta}-z\right|^{2}} d \tilde{\mu}(\theta), \quad|z|<1 .
$$

In the formulation of Theorem 2 we use the usual correspondence between $\mathbf{R}^{2}$ and the complex plane.

Theorem 2 is not entirely new. It appeared in a weaker form in [2; Theorem 2]. It was shown there that if $\left(4^{\prime}\right)$ holds, then there is a real number $t_{0}$ such that the limit $\left(1^{\prime}\right)$ exists for almost every real $t \in\left[t_{0}, t_{0}+2 \pi\right]$ with $I=I_{t}=\left\{e^{i \theta}: t_{0} \leqq \theta \leqq t\right\}$ and $\left(3^{\prime}\right)$ holds (provided the integral in $\left(3^{\prime}\right)$ is defined by integration by parts). In the proof of that theorem the authors made good use of a conformal mapping technique. The proof presented here seems to be more elementary, gives a stronger result, and generalizes to the higher dimensional case.

The author would like to express his deep gratitude to Boris Korenblum for his invaluable help in the course of the preparation of this paper.

\section{Definitions, notations and basic facts}

The Poisson kernel $\widetilde{P}$ has the following estimate, which is easily verified:

$$
\tilde{P}(r, t) \leqq 2\left(\frac{\pi}{t}\right)^{n}(1-r) \quad(0 \leqq r<1,0<t \leqq \pi) .
$$

For each $\eta \in S$ and each $t \in(0, \pi)$ the set $S_{\eta, t}=\{\xi \in S: \varphi(\eta, \xi)=t\}$ is an $(n-2)$ dimensional submanifold of $\mathbf{R}^{n}$. Denote by $\sigma_{\eta, t}$ the $(n-2)$-dimensional surface measure on $S_{\eta, t}$ so normalized that $\sigma_{\eta, t}\left(S_{\eta, t}\right)=1$. If $f$ is a nonnegative Borel measurable function on $S$, and $\eta \in S$ is arbitrary and fixed, then

$$
\int_{S} f d \sigma=\frac{1}{c_{1}} \int_{0}^{\pi}\left(\sin ^{n-2} t \int_{S_{\eta, t}} f d \sigma_{\eta, t}\right) d t
$$

where $c_{1}=\int_{0}^{\pi} \sin ^{n-2} t d t$. Therefore, for each fixed $x \in B, x \neq 0$, and $C(x)=$ $C(x /|x|, 1-|x|)$ we have

$$
\begin{gathered}
\inf _{\xi \in C(x)} P(x, \xi) \cdot \sigma(C(x))=\tilde{P}(|x|, 1-|x|) \cdot \frac{1}{c_{1}} \int_{0}^{1-|x|} \sin ^{n-2} t d t \\
\geqq \frac{1}{4 \pi^{n-2} c_{1}(n-1)}=c_{2},
\end{gathered}
$$

where the last inequality defines $c_{2}$. 
A subset $A$ of $S$ will be called a spherical simplex (or just a simplex) if $A$ can be written as the intersection of at most $n+1$ open caps. There exists a positive integer $N$ depending only on $n$ and with the following property: if a set $D \subset S$ is the intersection of a spherical simplex and a cap or the complement (in $S$ ) of a spherical simplex, then $D$ can be represented as the union of at most $N$ disjoint spherical simplices and a set of $\sigma$ measure zero. This becomes evident when we note that every open cap is the intersection of $S$ and an open half-space. For each $x \neq 0$ and $A \subset S, A \neq \emptyset$, we set $\varphi(x, A)=\inf _{\eta \in A} \varphi(x, \eta)$. Moreover, for each $A \subset S$, $\emptyset \neq A \neq S$, we denote $w(A)=\sup _{\eta \in A} \varphi(\eta, S \backslash A)$.

Let $k$ be a positive continuous nondecreasing unbounded function on $[0,1)$ such that $k(0)=0$ and

$$
J=J(k)=\int_{0}^{1}\left(\frac{k(r)}{1-r}\right)^{(n-1) / n} d r<+\infty .
$$

For each positive constant $M$ the condition

$$
k(\varrho(t))=M t^{-n}(1-\varrho(t)), \quad t \in(0, \pi),
$$

defines the unique function $\varrho=\varrho_{M}$ on $(0, \pi]$. If, in addition, we set $\varrho_{M}(0)=1$, then $\varrho_{M}$ will be a continuous decreasing function from $[0, \pi]$ into $(0,1]$. Since

we have

$$
\begin{aligned}
J^{n /(n-1)} & \geqq k(\varrho(t))\left(\int_{\varrho(t)}^{1}(1-r)^{(1-n) / n} d r\right)^{n /(n-1)} \\
& \geqq \frac{M}{(2 \pi)^{n}}\left(\frac{2 \pi(1-\varrho(t))}{t}\right)^{n /(n-1)},
\end{aligned}
$$

$$
1-\varrho(t) \leqq \frac{t}{2 \pi} \text { for each } t \in(0, \pi),
$$

whenever $M \geqq M_{0}=(2 \pi)^{n} J^{n /(n-1)}$. The last inequality will be assumed to hold in what follows. Let $0<t^{\prime}<t \leqq \pi$. Then

$$
\begin{gathered}
\int_{t^{\prime}}^{t} k(\varrho(\tau)) d \tau=M \int_{t^{\prime}}^{t}(1-\varrho(\tau)) d\left(\frac{\tau^{1-n}}{1-n}\right) \\
<\frac{M^{1 / n}}{n-1} \int_{\varrho(t)}^{\varrho\left(t^{\prime}\right)}\left[\left(\frac{k(r)}{1-r}\right)^{(n-1) / n}-\left(\frac{k(\varrho(t))}{1-\varrho(t)}\right)^{(n-1) / n}\right] d r+o(1) .
\end{gathered}
$$

By letting $t^{\prime}$ tend to 0 we obtain

$$
\int_{0}^{t} k(\varrho(\tau)) d \tau<\frac{M^{1 / n} \tilde{\varkappa}(t)}{n-1}
$$

where

$$
\tilde{\varkappa}(t)=\int_{\varrho_{M_{0}}(t)}^{1}\left(\frac{k(r)}{1-r}\right)^{(n-1) / n} d r, \quad t \in(0, \pi] .
$$

Note that the function $\tilde{x}$ is continuous and increasing, that $\tilde{x}(\pi) \leqq J$, and that $\lim _{t \rightarrow 0+} \tilde{\varkappa}(t)=0$. 


\section{Proof of Theorem 1}

For each $M \geqq M_{0}$ and each simplex $A \subset S, \emptyset \neq A \neq S$, we define an auxiliary mapping $\tilde{x}=\tilde{x}_{A, M}, \tilde{x}: S \rightarrow \bar{B}$, by the following formula:

$$
\tilde{x}(\eta)= \begin{cases}\eta, & \eta \in S \backslash A, \\ \varrho_{M}(\varphi(\eta, S \backslash A)) \eta, & \eta \in A .\end{cases}
$$

Let the auxiliary function $\tilde{v}=\tilde{v}_{A, M}$ be defined on $S$ by the following formula:

$$
\tilde{v}(\eta)= \begin{cases}0, & \eta \in S \backslash A, \\ k(|\tilde{x}(\eta)|), & \eta \in A\end{cases}
$$

We extend $\tilde{v}$ to the function $v=v_{A}, M$ defined on $\bar{B}$, by setting $v(x)=P[\tilde{v}](x)$ for $x \in B$. It is possible since we have the following

Lemma 1. The function $\tilde{v}_{A, M}$ is integrable with respect to $\sigma$. Moreover, there exists a constant $c_{3}>0$ depending only on $n$ such that

$$
\int \tilde{v}_{A, M} d \sigma \leqq c_{3} M^{1 / n} \tilde{\varkappa}(w(A)) \leqq c_{3} M^{1 / n} J .
$$

Proof of Lemma 1. Let $A=\bigcap_{j=1}^{l} C\left(\eta_{j}, t_{j}\right)$, where $l \leqq n+1, \eta_{j} \in S, t_{j} \in(0, \pi]$, $j=1,2, \ldots, l$. By the definition of $w(A)$, we have $A \subset \bigcup_{j=1}^{l} \tilde{C}_{j}$, where $\tilde{C}_{j}=$ $C\left(\eta_{j}, t_{j}\right) \backslash C\left(\eta_{j}, t_{j}-w(A)\right)$ if $t_{j}>w(A)$, and $\tilde{C}_{j}=C\left(\eta_{j}, t_{j}\right)$ if $t_{j} \leqq w(A), j=1,2, \ldots, l$. It is clear that $\tilde{v}_{A, M}(\eta) \leqq \max \left\{\tilde{v}_{C_{j}, M}(\eta): 1 \leqq j \leqq l\right\}$ for each $\eta \in S$. Therefore, if we denote $t_{j}^{\prime}=\max \left\{0, t_{j}-w(A)\right\}$, we will have:

$$
\begin{gathered}
\int \tilde{v}_{A, M} d \sigma \leqq \sum_{j=1}^{l} \int_{\tilde{c}_{j}} \tilde{v}_{c_{j}, M} d \sigma \\
=\sum_{j=1}^{l} \int_{\tilde{c}_{j}} k\left(\varrho_{M}\left(\varphi\left(\eta, S \backslash C_{j}\right)\right)\right) d \sigma(\eta) \\
=\sum_{j=1}^{l} \frac{1}{c_{1}} \int_{0}^{t_{j}-t_{j}^{\prime}} k\left(\varrho_{M}(t)\right) \sin ^{n-2} t d t \leqq \frac{(n+1) M^{1 / n}}{c_{1}(n-1)} \tilde{x}(w(A)),
\end{gathered}
$$

where the second equality follows from (8) and the last inequality from (12). This gives the first inequality of the lemma. The second one follows from the definition of $\tilde{\varkappa}$.

Lemma 2. There exists a constant $c_{4}>0$ depending only on $n$ such that for each simplex $A \subset S, \emptyset \neq A \neq S$, for each $M, M \geqq M_{0}$, and each $\eta \in A$ we have

$$
v_{A, M}(\tilde{x}(\eta)) \geqq c_{4} v_{A, M}(\eta) .
$$

Proof of Lemma 2. Note first that there exists a constant $c_{5}>0$ such that for arbitrary distinct points $\eta_{0}, \eta_{1} \in S$ and real numbers $t_{0}, t_{1}, 0<t_{0}<t_{1}=\varphi\left(\eta_{0}, \eta_{1}\right)$, we have

$$
\frac{\sigma\left(C_{0} \cap C_{1}\right)}{\sigma\left(C_{0}\right)} \geqq c_{5},
$$


where $C_{j}=C\left(\eta_{j}, t_{j}\right), j=0,1$. Now, fix $\eta_{0} \in A$. Let $\eta_{1} \in S \backslash A$ be such that $\varphi\left(\eta_{0}, \eta_{1}\right)=$ $\varphi\left(\eta_{0}, S \backslash A\right)$. Let $t_{1}=\varphi\left(\eta_{0}, \eta_{1}\right)$ and $t_{0}=1-\varrho_{M}\left(t_{1}\right)$. Note that, by (11), we have $0<t_{0}<t_{1}$. And, since $k\left(\varrho_{M}(t)\right)$ is a decreasing function of $t$, we have $v_{A, M}(\eta) \geqq$ $v_{A, M}\left(\eta_{0}\right)$ for each $\eta \in C_{1} \cap A$. Therefore

$$
v_{A, M}\left(\tilde{x}\left(\eta_{0}\right)\right) \geqq c_{5} \sigma\left(C_{0}\right) \cdot v_{A, M}\left(\eta_{0}\right) \cdot \inf _{\eta \in C_{0} \cap C_{1}} \tilde{P}\left(\tilde{x}\left(\eta_{0}\right), \eta\right) \geqq c_{5} c_{2} v_{A, M}\left(\eta_{0}\right),
$$

where the first inequality follows by (13) and the second by (9).

Now we are able to prove Lemma 3, which is an essential part of the proof of Theorem 1.

Lemma 3. There exists a constant $c_{6}>0$, depending only on $n$, such that for every function $u$, harmonic in a neighborhood of $\bar{B}$ with $u(0)=0$ and $u(x) \leqq k(|x|)$, $x \in B$, and for every spherical simplex $A \subset S, \emptyset \neq A \neq S$, we have

$$
\int_{A} u d \sigma \leqq c_{6} J^{1 /(n-1)} \tilde{\varkappa}(w(A)) .
$$

Proof of Lemma 3. Let $\mathscr{A}$ be the family of all simplices in $S$. Let us denote $K=\sup _{A \in \mathscr{A}} \int_{A} u d \sigma$. Note that, since $\int_{S} u d \sigma=0$, we have $\int_{A} u d \sigma \geqq-N K$ and $\int_{C \backslash A} u d \sigma \geqq-N^{3} K$ for arbitrary $A \in \mathscr{A}$ and an arbitrary cap $C$, where $N$ is the constant from the previous section. Now, for arbitrary $A \in \mathscr{A}, \emptyset \neq A \neq S$, and $x \in B$, $x \neq 0$, such that $\varphi(x, S \backslash A)=t_{0}>0$, we have

$$
P\left[u \cdot \mathbf{1}_{S \backslash A}\right](x)=\int_{t_{0}}^{\pi} \tilde{P}(|x|, t) d\left(\int_{C(x|| x \mid, t) \backslash A} u d \sigma\right) \geqq \tilde{P}\left(|x|, t_{0}\right)\left(-N^{3} K\right) .
$$

Observe that (7), (10) and what is above give

$$
P\left[u \cdot \mathbf{1}_{S \backslash A}\right](\tilde{x}(\eta)) \geqq-\frac{c_{7} K}{M} k(|\tilde{x}(\eta)|)
$$

for each $\eta \in A$, where $c_{7}=4 \pi^{n} N^{3}$.

For each $A \in \mathscr{A}$ and each $M \geqq M_{0}$ set

$$
h=h_{A, M}=u \cdot \mathbf{1}_{A}-\frac{1}{c_{4}}\left(\frac{c_{7} K}{M}+1\right) \tilde{v}_{A, M},
$$

and extend $h$ to the whole of $\bar{B}$ by its Poisson integral. Then by Lemma 1 we have

$$
h(0) \geqq \int_{A} u d \sigma-\frac{c_{3}}{c_{4}}\left(\frac{c_{7} K}{M}+1\right) M^{1 / n} \tilde{\varkappa}(w(A)) .
$$

On the other hand, $u \cdot \mathbf{1}_{A}=u-\mathbf{1}_{S \backslash A} \cdot u$ on $S$, and hence, by Lemma 2 and (14), we have

$$
h(\tilde{x}(\eta)) \leqq 0 \quad \text { for every } \eta \in A .
$$

Let $G=\{x \in \bar{B}: x \neq 0,|x|<\tilde{x}(x /|x|)\} \cup\{0\}$. We can write $\partial G=G_{1} \cup G_{2} \cup G_{3}$, where $G_{1}=\tilde{x}(A), G_{2}$ is the interior (in the relative topology on $S$ ) of $S \backslash A$ and $G_{3}$ is the boundary of $A$ (also in the relative topology on $S$ ). The function $h$ is har- 
monic on $G$ and continuous on $\bar{G} \backslash G_{3}$. Moreover, $h(\eta)=0$ for each $\eta \in G_{2}$ by the definition of $h$, and, by (16), $h \leqq 0$ on $G_{1}$. Since $h$ is bounded from above on $B \supset G$, and since $G_{3}$ is a polar set, we have, by a version of the maximum principle due to Ascoli ([1], Theorem 5.16), that $h \leqq 0$ on $G$. In particular $h(0) \leqq 0$. Therefore, by (15), we have

$$
\int_{A} u d \sigma \leqq \frac{c_{3}}{c_{4}}\left(\frac{c_{7} K}{M}+1\right) M^{1 / n} \tilde{\chi}(w(A)),
$$

and, consequently,

$$
K \leqq \frac{c_{3}}{c_{4}}\left(\frac{c_{7} K}{M}+1\right) M^{1 / n} J .
$$

Since the last inequality holds for each $M \geqq M_{0}=(2 \pi)^{n} J^{n /(n-1)}$, there is a constant $c_{8}$ depending only on $n$ such that $K \leqq c_{8} J^{1 /(n-1)}$. This together with (17) implies the lemma.

Now, let $x$ be an arbitrary continuous increasing function on $[0, \pi]$ with $\varkappa(0)=0$. A real function $f$ defined on $[0, \pi]$ is said (in this section only) to have the $x$-property if $f(0)=f(\pi)=0, f\left(t_{2}\right)-f\left(t_{1}\right) \leqq \varkappa\left(t_{2}-t_{1}\right)$ whenever $0 \leqq t_{1}<t_{2} \leqq \pi$, and $f(t)=[f(t+)+f(t-)] / 2$ whenever it makes sense. Note that if $f$ has the $x$-property, it has one-sided limits at each $t \in[0, \pi]$ (so the last condition for the $x$-property is satisfied at each $t \in(0, \pi))$ and, consequently, $f$ has at most a countable number of points of discontinuity, and that $|f(t)| \leqq \varkappa(\pi)$ for each $t \in[0, \pi]$. In the next step of the proof of Theorem 1 we will need the following Helly-type selection theorem adopted from [4] (Theorem 1, p. 204).

Lemma 4. Let $x$ be a function as above. Let $\left\{f_{j}\right\}_{j=1}^{\infty}$ be a sequence of real functions such that each $f_{j}$ is defined on $[0, \pi]$ and has the $x$-property. Then there exist a function $f$ on $[0, \pi]$ having the $x$-property and a subsequence $\left\{f_{j_{1}}\right\} \quad\left(j_{1}<j_{2}<\ldots\right)$ which converges to $f$ at every point of continuity of $f$.

The proof of Lemma 4 can be established by a slight variation of the classical proof of the Helly selection theorem (cf. [3]).

Now, assume that $u$ satisfies the assumptions of Theorem 1 . Note first that it is sufficient to prove the theorem in the case where $u(0)=0$. Moreover, we can and do assume that $u$ is unbounded from above, since otherwise the result is classical. Now, set $k(r)=m(r), 0 \leqq r<1$. Such a $k$ has all the properties that we assumed for the function $k$ introduced in the second section. Let $u^{(r)}(x)=u(r x)$ for each $r \in(0,1)$ and $x \in B / r$. Fix an arbitrary $\eta \in S$, and set

$$
u_{\eta}^{(r)}(t)=\int_{C(\eta, t)} u^{(r)} d \sigma, \quad 0 \leqq t \leqq \pi .
$$

Since, $u^{(r)}(x) \leqq k(|r x|) \leqq k(|x|), r \in(0,1), x \in B$, Lemma 3 gives

$$
u^{(r)}\left(t_{2}\right)-u^{(r)}\left(t_{1}\right)=\int_{\overline{C\left(\eta, t_{2}\right) \backslash \tau\left(\eta, t_{1}\right)}} u^{(r)} d \sigma \leqq c_{6} J^{1 /(n-1)} \tilde{\varkappa}\left(t_{2}-t_{1}\right),
$$


whenever $0 \leqq t_{1}<t_{2} \leqq \pi$, because $w\left(\bar{C}\left(\eta, t_{2}\right) \backslash \bar{C}^{\prime}\left(\eta, t_{1}\right)\right)=\left(t_{2}-t_{1}\right) / 2$. Let us denote $\chi(t)=c_{6} J^{1 /(n-1)} \tilde{\chi}(t)$. Observe that $\chi$ as a function on $[0, \pi]$ is continuous and increasing, $\chi(0)=0$, and $\chi(\pi) \leqq c_{6} J^{n /(n-1)}$. From the inequality (18) it follows that each $u_{\eta}^{(r)}$ has the $x$-property. Lemma 4 implies that for each $\eta \in S$ there exist a sequence $\left\{r_{j}\right\}_{j=1}^{\infty}, 0<r_{j}<1$, tending to 1 , and a function $f_{\eta}$ on $[0, \pi]$ having the $\varkappa$-property such that $\lim _{j \rightarrow \infty} u_{\eta}^{\left(r_{j}\right)}(t)=f_{\eta}(t)$, whenever $t$ is a point of continuity of $f$. Now, for each $x \in B$ such that $x /|x|=\eta$ we have

$$
\begin{aligned}
u(x) & =\lim _{j \rightarrow \infty} u\left(r_{j} x\right)=\lim _{j \rightarrow \infty} \int_{0}^{\pi} \tilde{P}(|x|, t) d u_{\eta}^{\left(r_{j}\right)}(t) \\
& =-\lim _{j \rightarrow \infty} \int_{0}^{\pi} u_{\eta}^{\left(r_{j}\right)}(t) \frac{\partial}{\partial t} \tilde{P}(|x|, t) d t \\
& =-\int_{0}^{\pi} f_{\eta}(t) \frac{\partial}{\partial t} \tilde{P}(|x|, t) d t=\int_{0}^{\pi} \tilde{P}(|x|, t) d f_{\eta}(t),
\end{aligned}
$$

by integration by parts and the Lebesgue dominated convergence theorem.

Now, it remains to prove that the limit $u_{\eta}(t)$ exists for each $t \in[0, \pi]$ and $u_{\eta}=f_{\eta}$. For this purpose let us state an elementary lemma.

Lemma 5. For each $r \in(0,1)$ and $t \in(0, \pi)$ let us define a function $p_{t}^{(r)}$ on $[0, \pi]$ writing $p_{t}^{(r)}(s)=P\left[\mathbf{1}_{C(\eta, t)}\right](r \zeta)$, where $\zeta$ is an arbitrary element of $S$ satisfying the relation $\varphi(\eta, \zeta)=s$. Then $p_{t}^{(r)}$ is a well-defined continuously differentiable, decreasing function on $[0, \pi]$, and for each $t \in(0, \pi)$ we have

$$
\lim _{r \rightarrow 1-} p_{t}^{(r)}(s)=\left\{\begin{array}{lll}
1 & \text { if } & 0 \leqq s<t \\
\frac{1}{2} & \text { if } & s=t \\
0 & \text { if } & t<s \leqq \pi
\end{array}\right.
$$

Moreover, if $g$ is a harmonic function on a neighborhood of $\bar{B}$, then for each $\eta \in S$, $t \in(0, \pi), r \in(0,1)$ we have

$$
\int_{\bar{C}(\eta, t)} g(r \zeta) d \sigma(\zeta)=\int_{0}^{\pi} p_{t}^{(r)}(s) d\left(\int_{\bar{C}(\eta, s)} g(\zeta) d \sigma(\zeta)\right) .
$$

Using this lemma, the integration-by-parts formula and the Lebesgue dominated convergence theorem, we obtain first, for arbitrary $r \in(0,1)$ and $t \in(0, \pi)$,

$$
\begin{aligned}
& \int_{C(\eta, t)} u^{(r)} d \sigma=\lim _{j \rightarrow \infty} \int_{C(\eta, t)} u^{\left(r \cdot r_{j}\right)} d \sigma \\
& =-\lim _{j \rightarrow \infty} \int_{0}^{\pi} u^{\left(r_{j}\right)}(s) \frac{\partial}{\partial s} p_{t}^{(r)}(s) d s \\
& =-\int_{0}^{\pi} f_{\eta}(s) \frac{\partial}{\partial s} p_{t}^{(r)}(s) d s .
\end{aligned}
$$


Then, by letting $r$ tend to 1 above, we get (again by Lemma 5):

$$
\lim _{r \rightarrow 1-} \int_{C(\eta, t)} u^{(r)} d \sigma=\frac{f_{\eta}(t-)+f_{\eta}(t+)}{2} .
$$

This concludes the proof of Theorem 1 .

\title{
4. Conclusion
}

Theorem 2 is, apart from unessential changes, a special case of Theorem 1. On the other hand, the method used in the proof of Theorem 1 is greatly simplified when used to prove its 2-dimensional version, Theorem 2. The reason for this is that the concept of spherical simplices can then be replaced by that of circular arcs. It should be noted that the last part of the proof of this case is essentially contained in [4] (Theorem 2, p. 212).

It is obvious that using translations and dilations we can generalize our result to the case of an arbitrary open ball in $\mathbf{R}^{n}$. Furthermore, employing also the Kelvin transform we can obtain from our theorems their half-space (in the case of Theorem 2, "half-plane") analogues.

\section{References}

[1] Hayman, W. K., and P. B. Kennedy: Subharmonic Functions, Vol. I. - London Mathematical Society Monographs, No 9. Academic Press, London-New York, 1976.

[2] Hayman, W. K., and B. Korenblum: An extension of the Riesz-Herglotz formula. - Ann. Acad. Sci. Fenn. Ser. A I Math. 2, 1976, 175-201.

[3] Helly, E.: Über lineare Functionaloperationen. - Wien. Ber. 121, 1912, 265-297.

[4] Korenblum, B.: An extension of the Nevanlinna theory. - Acta Math. 135, 1975, 187-219.

\author{
Technical University \\ Department of Mathematics \\ Wybrzeże Wyspiańskiego 27 \\ 50-370 Wrocław \\ Poland
}

Received 12 March 1984 